\title{
Gestação após gastroplastia para tratamento de obesidade mórbida: série de casos e revisão da literatura
}

\author{
Pregnancy after gastroplasty for treatment of morbid obesity: a case series and review of the literature \\ Josivan Gomes de Lima ${ }^{1}$, Lúcia Helena Coelho Nóbrega ${ }^{2}$, Juliana Bezerra Mesquita ${ }^{3}$, Maria Lúcia Coelho Nóbrega ${ }^{4}$, \\ Aldo da Cunha Medeiros ${ }^{5}$, Tecia Maria de Oliveira Maranhão ${ }^{6}$, George Dantas Azevedo ${ }^{7}$
}

\section{RESUMO}

Objetivo: relatar a evolução de uma série de casos de gestação em mulheres previamente submetidas à cirurgia de bypass gástrico para tratamento de obesidade grave. Métodos: cinco casos consecutivos de gravidez após gastroplastia ocorridos entre 2001 e 2004 foram avaliados. As pacientes tinham idade entre 30 e 34 anos e todas haviam sido submetidas à cirurgia de Capella. Aspectos clínicos, laboratoriais e do acompanhamento materno e fetal foram considerados, durante o período gestacional e após o parto. Foi realizada revisão da literatura internacional, por meio das bases de dados MEDLINE e Web of Science, utilizando os seguintes unitermos: gastroplasty, gastric bypass surgery, bariatric surgery e pregnancy. Resultados: todas as gestações observadas foram únicas e não ocorreram complicações obstétricas, durante o seguimento pré-natal e parto. Também não houve registro de recém-nascidos prematuros ou de baixo peso ao nascimento. Conclusão: nossos dados sugerem que a gravidez após gastroplastia é segura para a mãe e feto. Entretanto, em virtude do limitado volume de informação disponível sobre o tema, investigações adicionais são necessárias para estabelecer recomendações apropriadas com relação ao seguimento dessas gestações.

PALAVRAS-CHAVE: Gastroplastia; Obesidade mórbida/cirurgia; Gravidez; Obesidade mórbida

\section{ABSTRACT}

Purpose: we report a small series of pregnant women who underwent gastric bypass surgery for severe obesity, with a review of the literature on this topic. Methods: five consecutive cases of pregnancy after gastroplasty between 2001 and 2004 were evaluated, and clinical, laboratory and therapeutic features were considered. Patients were 30 to 34 years old and all had been submitted to gastroplasty by the Capella technique. The outcomes for both the pregnant woman and the fetus were evaluated. A search of the English language literature was done through MEDLINE and Web of Science databases with the following terms: gastroplasty, gastric bypass surgery, bariatric surgery, and pregnancy. Results: all 5 pregnancies were singleton. No major obstetric complications were observed and there were no premature or lowbirth weight infants. Conclusion: our data suggest that pregnancy following gastroplasty is safe for mother and fetus. However, since information about this topic is limited, further investigations are required to establish appropriate recommendations concerning the follow-up of these pregnancies.

KEYWORDS: Gastroplasty; Obesity, morbid/surgery; Pregnancy; Obesity, morbid

\footnotetext{
Instituição: Programa de Pós-graduação em Ciências da Saúde (PPGCSA) - Centro de Ciências da Saúde - Universidade Federal do Rio Grande do Norte UFRN - Natal (RN) - Brasil

1 Professor do Departamento de Medicina Clínica (Endocrinologia) e mestrando do PPGCSA - Universidade Federal do Rio Grande do Norte - UFRN Natal (RN), Brasil.

2 Médica Endocrinologista do Hospital Universitário Onofre Lopes e mestranda do PPGCSA - Universidade Federal do Rio Grande do Norte - UFRN - Natal (RN), Brasil.

3 Acadêmica do Curso de Medicina - Universidade Federal do Rio Grande do Norte - UFRN - Natal (RN), Brasil.

4 Médica Endocrinologista - Centro de Endocrinologia de Natal - Natal (RN), Brasil.

5 Professor do Departamento de Cirurgia e Orientador do PPGCSA - Universidade Federal do Rio Grande do Norte - UFRN - Natal (RN), Brasil.

6 Professora do Departamento de Tocoginecologia e Orientador do PPGCSA - Universidade Federal do Rio Grande do Norte - UFRN - Natal (RN), Brasil.

7 Professor do Departamento de Morfologia e Coordenador do PPGCSA - Centro de Biociências - Universidade Federal do Rio Grande do Norte - UFRN Natal (RN), Brasil

Correspondence: George Dantas de Azevedo

Departamento de Morfologia do Centro de Biociências - Campus Universitário, BR 101 - Lagoa Nova - 59078-970 - Natal - RN - Telefone: (84) 32153431- Fax: (84) 32119207 - e-mail: georgedantas@uol.com.br
} 


\section{Introdução}

A obesidade é considerada atualmente uma epidemia mundial, sendo as diversas formas de tratamento disponíveis, na maioria das vezes, ineficazes em longo $\mathrm{prazo}^{1,2}$. Nos casos de obesidade mórbida (definida como índice de massa corpórea (IMC) maior que $40 \mathrm{~kg} / \mathrm{m}^{2}$ ), o tratamento apresenta resultados ainda mais desanimadores.

A cirurgia bariátrica surgiu como opção terapêutica para a obesidade mórbida, mostrandose eficaz em curto e longo prazo, não somente no que diz respeito à perda de peso, mas também à melhora e até resolução de comorbidades como diabete melito e hipertensão arterial, entre ou$\operatorname{tras}^{3}$.

$\mathrm{Na}$ atualidade, cerca de $84 \%$ dos pacientes que se submetem à gastroplastia são mulheres, muitas delas em idade fértil ${ }^{4}$. Nesse sentido, vislumbram-se beneficios adicionais da cirurgia, relacionados à esfera reprodutiva, tais como aumento da atividade sexual ${ }^{5}$ e melhora significativa na fertilidade $^{6}$, a despeito da possibilidade de essas pacientes poderem evoluir com má nutrição induzida pela cirurgia ${ }^{7}$. Considerando esse último aspecto e a realidade de que muitas dessas mulheres podem vir a engravidar após a gastroplastia, especula-se o quanto a gestação poderia evoluir com resultados desfavoráveis nessa situação em particular.

A literatura é ainda deficiente de informações sobre os resultados de gravidez após realização de gastroplastia, havendo opiniões contraditórias advindas, na maioria das vezes, de relatos de casos e estudos com casuística reduzida. No presente artigo, os autores relatam resultados de uma série de casos de gestações em pacientes previamente submetidas à gastroplastia, enfocando especificamente os resultados obstétricos e perinatais.

\section{Métodos}

Foi realizado estudo retrospectivo constando de análise dos prontuários médicos de pacientes do sexo feminino submetidas à cirurgia bariátrica e acompanhadas clinicamente, no período de 2001 a 2004. A partir dessa análise inicial, foram detectadas cinco gestações ocorridas após a realização da cirurgia, as quais constituíram o objeto da análise. O estudo insere-se no contexto de uma pesquisa mais ampla destinada a avaliar os efeitos da obesidade mórbida sobre a função reprodutiva. O protocolo experimental foi analisado e aprovado pelo Comitê de Ética em Pesquisa da Universidade Federal do Rio Grande do Norte.

A técnica cirúrgica empregada em todos os casos foi a de Capella, que consiste na realização de gastroplastia com Y de Roux, pela qual se deixa uma pequena bolsa gástrica que, isolada do restante do estômago, esvazia diretamente na alça do intestino delgado.

Foi empregado protocolo de pesquisa especificamente elaborado para obter informações sobre as variáveis relacionadas aos dados antropométricos maternos, no pré e pós-operatório da gastroplastia, parâmetros clínicos e ultra-sonográficos de acompanhamento pré-natal, resolução da gestação e dados perinatais. As principais variáveis consideradas para análise foram: IMC prévio à gastroplastia e por ocasião do diagnóstico de gestação, características do ciclo menstrual pré e pósgastroplastia, comorbidades associadas à obesidade e intercorrências clínicas no pós-operatório.

Uma revisão da literatura científica internacional sobre o tema foi realizada, por meio das bases de dados MEDLINE e Web of Science, utilizando-se cruzamentos dos seguintes unitermos: gastroplasty, gastric bypass surgery, bariatric surgery e pregnancy.

\section{Resultados}

Na Tabela 1 são sumarizados os resultados relacionados ao IMC e perfil menstrual das pacientes, antes e após a gastroplastia. Observa-se que as pacientes obtiveram redução considerável de peso após a cirurgia e que houve regularização do ciclo menstrual na única paciente que apresentava quadro de amenorréia, prévio à gastroplastia. Nenhuma das pacientes necessitou da utilização de medicamentos para indução de ovulação, sendo as cincos gestações espontâneas. Durante o seguimento pré-natal, todas utilizaram reposição de ferro e polivitamínicos, em doses habituais.

Em relação aos resultados perinatais, a Tabela 1 mostra que as cinco gestações foram únicas e foram resolvidas no termo da gravidez. Não houve registro de baixo peso ao nascimento e nenhum recém-nascido necessitou de cuidados intensivos no pós-parto imediato ou tardio. Até o momento da submissão desse artigo, as crianças se encontravam sem anormalidades no desenvolvimento neuromotor, com média de acompanhamento de 13 meses. 
Tabela 1 - Resultados maternos e fetais, antes e após realização da gastroplastia.

\begin{tabular}{|c|c|c|c|c|c|}
\hline & Caso 1 & Caso 2 & Caso 3 & Caso 4 & Caso 5 \\
\hline Idade no momento da gestação & 31 anos & 30 anos & 33 anos & 30 anos & 34 anos \\
\hline Tipo de cirurgia & Capella aberta & Capella aberta & Capella laparoscópica & Capella aberta & Capella aberta \\
\hline IMC quando engravidou & $29,5 \mathrm{~kg} / \mathrm{m}^{2}$ & $27,0 \mathrm{~kg} / \mathrm{m}^{2}$ & $31,1 \mathrm{~kg} / \mathrm{m}^{2}$ & $26,8 \mathrm{~kg} / \mathrm{m}^{2}$ & $31,1 \mathrm{~kg} / \mathrm{m}^{2}$ \\
\hline Tempo entre a cirurgia e o início da gestação & 8 meses & 20 meses & 14 meses & 29 meses & 36 meses \\
\hline Duração da gestação & 39 semanas & 39 semanas & 38 semanas & 38 semanas & 38 semanas \\
\hline Tipo de parto & Cesáreo & Normal & Cesáreo & Cesáreo & Cesáreo \\
\hline Peso/estatura fetais & $2962 \mathrm{~g} / 48 \mathrm{~cm}$ & $3715 \mathrm{~g} / 50 \mathrm{~cm}$ & $3000 \mathrm{~g} / 48 \mathrm{~cm}$ & $2775 \mathrm{~g} / 47 \mathrm{~cm}$ & $3250 \mathrm{~g} / 52 \mathrm{~cm}$ \\
\hline Apgar (1/5 minutos) & $3 / 8$ & $7 / 9$ & $9 / 9$ & $9 / 10$ & $N D^{*}$ \\
\hline Complicações fetais & Nenhuma & Nenhuma & Nenhuma & Nenhuma & Nenhuma \\
\hline
\end{tabular}

ND: não disponível; IMC = índice de massa corporal.

A seguir, apresentamos descrições simplificadas dos aspectos clínicos e obstétricos de cada caso em particular.

\section{Caso 1}

Paciente de 31 anos tinha IMC de 47,5 kg/ $\mathrm{m}^{2}$ antes da cirurgia; era hipertensa controlada com medicação. Os ciclos menstruais pré e póscirurgia eram regulares. Antecedente de duas gestações prévias, com um aborto espontâneo. Engravidou espontaneamente, após oito meses da gastroplastia, quando estava com IMC de 29,5 kg/ $\mathrm{m}^{2}$. Com 14 semanas de gestação, apresentou quadro de ameaça de abortamento, que foi controlado com repouso, uso de antiespasmódicos e suplementação de progesterona natural $(300 \mathrm{mg} /$ dia). Durante a gestação, os exames laboratoriais demonstraram niveis de calcemia $(9,1 \mathrm{mg} / \mathrm{dL})$ e albumina $(3,3 \mathrm{mg} / \mathrm{dL})$ nos limites inferiores da normalidade, sendo feitas suplementações de carbonato de cálcio (500 mg, 2 vezes / dia) e suplemento protéico. A resolução da gestação ocorreu na 39a semana, por parto cesáreo, sem intercorrências. Recém-nascido saudável, com peso de 2.962 g, estatura de $48 \mathrm{~cm}$ e Apgar 3/8.

\section{Caso 2}

Paciente de 30 anos, ciclos menstruais regulares pré e pós-cirurgia. O IMC prévio à gastroplastia era de $50 \mathrm{~kg} / \mathrm{m}^{2}$. Evoluiu no pós-operatório com anemia, que foi tratada com suplementação de ferro. Cerca de 20 meses após a cirurgia, engravidou espontaneamente, quando estava com $\mathrm{IMC}=27,0 \mathrm{~kg} / \mathrm{m}^{2}$. Não houve intercorrências na gestação e a resolução se deu por parto normal, a termo. Recém-nascido saudável, com peso de 3.715 $\mathrm{g}$, estatura de $50 \mathrm{~cm}$ e Apgar 7/9.

\section{Caso 3}

Paciente de 33 anos, obesa mórbida $\left(\mathrm{IMC}=52,4 \mathrm{~kg} / \mathrm{m}^{2}\right)$, com antecedente de irregularidade menstrual do tipo oligoamenorréia, desde a menarca, só ocorrendo menstruação após uso oral de acetato de medroxiprogesterona. Após a cirurgia evoluiu com regularização dos ciclos menstruais, sem uso de medicação e engravidou espontaneamente após 14 meses $\left(\mathrm{IMC}=31,1 \mathrm{~kg} / \mathrm{m}^{2}\right)$. A gestação transcorreu sem anormalidades com resolução na $38^{\mathrm{a}}$ semana, por cesárea, sem intercorrências no parto e puerpério imediato. Recémnascido saudável, com peso de $3.000 \mathrm{~g}$, estatura de $48 \mathrm{~cm}$ e índice Apgar 9/9.

\section{Caso 4}

Paciente 30 anos, IMC $=45,4 \mathrm{~kg} / \mathrm{m}^{2}$, hipertensa não controlada desde a sua última gestação, aos 26 anos, quando teve pré-eclâmpsia. Ciclos menstruais regulares antes e após a cirurgia. Antecedente de tiroidectomia devido a carcinoma papilífero. Foi submetida à cirurgia bariátrica pela técnica de Capella aberta; evoluiu no pós-operatório imediato com hemorragia interna, sendo administrados cinco bolsas de sangue. Engravidou, espontaneamente, 29 meses após a cirurgia, com IMC $=26,8 \mathrm{~kg} / \mathrm{m}^{2}$. Apresentou anemia, sendo tratada com suplementação de ferro. Com 35 semanas de idade gestacional, apresentou aumento da pressão arterial e foi administrado metildopa. A resolução da gestação ocorreu na $38^{a}$ semana, por cesárea, sem intercorrências. Recém-nascido saudável, com peso de 2.775 g, estatura de $47 \mathrm{~cm}$ e Apgar 9/10.

\section{Caso 5}

Paciente de 30 anos tinha $\mathrm{IMC}=50,45 \mathrm{~kg} /$ $\mathrm{m}^{2}$ antes da cirurgia, menarca aos 13 anos e ci- 
clos menstruais pré e pós-cirurgia regulares. Engravidou espontaneamente, após três anos da gastroplastia, quando estava $\operatorname{com} \mathrm{IMC}=31,1 \mathrm{~kg} /$ $\mathrm{m}^{2}$. Fez acompanhamento obstétrico e tomou vitaminas usuais da gestação, evoluindo sem complicações. A resolução da gestação ocorreu na $38^{a}$ semana, por parto cesáreo, sem intercorrências. Recém-nascido saudável, com peso de 3.250 g, estatura de $52 \mathrm{~cm}$.

\section{Discussão}

A obesidade acarreta, reconhecidamente, problemas para a fertilidade feminina. Apesar de a maioria das portadoras de obesidade grave menstruar regularmente ${ }^{8}$, não pode se afirmar que a ovulação ocorre regularmente nessas mulheres, de forma que a infertilidade pode representar condição freqüentemente associada ao quadro de obesidade. A gravidez, quando ocorre, é considerada de alto risco, havendo maior incidência de diabete gestacional, pré-eclâmpsia e resolução do parto por cesariana, além dos riscos inerentes ao feto, como macrossomia e hipoglicemia neonatal ${ }^{9}$.

Por induzir perda de peso e atenuar riscos inerentes às comorbidades associadas à obesidade mórbida, a cirurgia bariátrica apresenta potencial também para ocasionar beneficios relacionados à esfera reprodutiva, tanto no homem, quanto na mulher. A despeito disso, em decorrência das alterações nutricionais determinadas pelo bypass gástrico, especula-se o quanto a gestação ocorrida após gastroplastia estaria associada com maior risco materno e fetal.

A literatura ainda é deficiente de informações definitivas sobre o tema, sendo a maioria dos estudos publicados referentes a relatos de casos ou pequenas casuísticas acompanhadas. Muitos relatos são feitos apenas quando os resultados obstétricos são adversos, dando a falsa impressão de que estes são muito freqüentes ${ }^{10}$. No presente artigo, os autores relatam os resultados obstétricos e perinatais de cinco gestações ocorridas após importante perda de peso induzida pela cirurgia bariátrica, com o objetivo de contribuir para o conhecimento científico nessa área e fornecer subsídios para a obtenção de recomendações apropriadas ao seguimento dessas gestações.

A técnica de Capella é uma das técnicas mais utilizadas em todo o mundo ${ }^{4}$ e com bons resultados em curto e longo prazo $^{3}$. As cinco pacientes relatadas foram submetidas à mesma técnica, diferindo apenas na via de acesso cirúrgico. Ao nosso ver, esse aspecto não parece acarretar interferência significativa para os resultados de gestação, alertando-se apenas para o fato de que, no caso da cirurgia aberta, deve ser respeitado periodo de tempo suficiente para haver a completa cicatrização da parede abdominal, prévio à gravidez, conforme recomendado em relação a qualquer laparotomia.

Em relação ao padrão menstrual, em estudo prévio relatamos que a maioria das pacientes obesas ainda menstrua regularmente ${ }^{8}$, não significando obrigatoriamente que ovulam. Após a cirurgia bariátrica, aquelas que apresentam alguma irregularidade menstrual geralmente passam a ter ciclos regulares, conforme aconteceu na paciente do caso 3. Devemos orientar as pacientes que, caso não queiram engravidar, devem ter muito mais cuidado após a perda de peso e sugerir o melhor método anticoncepcional, evitando os anticoncepcionais orais devido à possibilidade de absorção errática. Uma das pacientes aqui relatadas teve a gestação não planejada, só descoberta no $3^{\circ}$ mês.

Em nossa pequena série de casos, não foram evidenciadas complicações maternas nem fetais dignas de nota, corroborando achados de outros autores $^{5,6,11}$. Dados da literatura apontam que, comparando-se gestações de pacientes obesas mórbidas antes da gastroplastia e após a perda de peso induzida pela cirurgia, encontra-se redução significativa na necessidade de cesárea, incidência de macrossomia e diabete gestacional ${ }^{5,12}$.

Fato particularmente importante em relação ao planejamento da gestação em pacientes previamente submetidas à gastroplastia diz respeito aos aspectos nutricionais. Uma vez que essas pacientes podem evoluir com deficiências de alguns compostos como ferro, vitamina $B_{12}$, folato e cálcio, isso poderia resultar em aumento do risco para complicações maternas (anemia) e fetais (defeito no tubo neural, restrição no crescimento intra-uterino), recomendando-se o adequado manejo nutricional no pós-operatório, especialmente quando se planeja futura gestação, para assegurar concentrações séricas adequadas desses nutrientes antes da gestação.

É rotina em nosso serviço a utilização crônica de compostos polivitamínicos para todos os pacientes que realizaram gastroplastia. Os acompanhamentos são usualmente semestrais, e se o paciente apresenta anemia, deficiência de albumina ou hiperparatiroidismo secundário, suplementação de sulfato ferroso, proteínas e cálcio/vitamina $\mathrm{D}$, respectivamente, são iniciadas. Apesar de nenhuma das pacientes ter realizado acompanhamento nutricional com o objetivo préconcepcional, nenhuma delas apresentou quadro de desnutrição durante a gestação.

Anemia, quadro freqüente na gestação ${ }^{13}$, seja por deficiência de ferro, ácido fólico ou vitamina $B_{12}$, foi encontrada apenas na paciente 4 
durante a gestação. Saliente-se o fato de que todas utilizaram reposição de sulfato ferroso, conforme a rotina padronizada de acompanhamento pré-natal. Essa pequena incidência de anemia também foi citada por Wittgrove et al. ${ }^{12}$. Ocorrência de hérnias internas tem sido relatada na literatura, de modo que devemos ficar atentos a essa complicação potencial ${ }^{10,14}$.

Alguns cuidados devem ser levados em consideração para minimizar os riscos da gestação após gastroplastia. A paciente deve ser aconselhada a não engravidar no primeiro ano pós-cirurgia, visto que esse é o período em que a perda de peso se faz de forma mais intensa. Se a paciente engravida nesses primeiros doze meses, certamente perderá a fase de maior perda ponderal, e a potencial perda de peso provavelmente não será retomada após o parto. Além disso, as quantidades de alimentos ingeridos nessa fase de adaptação geralmente são menores, de modo que o risco de desnutrição, teoricamente, é maior. A despeito dos aspectos destacados, ilustre-se o fato de que uma das pacientes relatadas engravidou no $8^{\circ}$ mês pós-cirurgia, sem ocorrência de complicações maternas ou fetais.

Outro aspecto importante do acompanhamento pré-natal, que merece consideração especial nas mulheres que engravidam após gastroplastia, diz respeito ao rastreamento do diabete gestacional. Essa estratégia é comumente realizada pelo do teste oral de tolerância à glicose, após sobrecarga oral de $50 \mathrm{~g}, 75 \mathrm{~g}$ ou $100 \mathrm{~g}$ de glicose, entre a $24^{a}$ e a $28^{a}$ semana de gestação. A realização do teste oral de tolerância à glicose não deve ser recomendada nessas pacientes, tendo em vista o risco do aparecimento da sindrome de dumping. Essa sindrome decorre do esvaziamento gástrico rápido e consiste de náuseas, vômitos, sudorese fria, astenia, desconforto abdominal, etc.

A análise dos resultados obstétricos e perinatais da pequena série de casos relatada sugere que a gestação após gastroplastia é segura para o binômio materno-fetal. Nosso estudo reforça resultados de outros autores demonstrando que, apesar de possivel desnutrição materna induzida pela gastroplastia ${ }^{5,7}$, a cirurgia bariátrica aparentemente não compromete o desenvolvimento fetal intra-uterino nem o crescimento e desenvolvimento da criança no primeiro ano de vida. Entretanto, como todo estudo dessa natureza, nosso trabalho apresenta limitações relacionadas, principalmente, ao número de pacientes estudadas e à análise de apenas um tipo de técnica. Diante disso, investigações adicionais são necessárias para estabelecer recomendações apropriadas com relação ao seguimento dessas gestações.

\section{Agradecimentos}

A equipe de cirurgióes da UNICAD - Unidade de Nutrição e Cirurgia do Aparelho Digestivo e aos médicos obstetras responsáveis pelo atendimento pré-natal das pacientes incluidas no estudo.

\section{Referências}

1. Skidmore PM, Yarnell JW. The obesity epidemic: prospects for prevention. QJM. 2004;97(12):817-25.

2. Wooley SC, Garner DM. Dietary treatments for obesity are ineffective. BMJ.1994;309(6955):655-6.

3. Sjostrom L, Lindroos AK, Peltonen M, Torgerson J, Bouchard C, Carlsson B, et al. Lifestyle, diabetes, and cardiovascular risk factors 10 years after bariatric surgery. N Engl J Med. 2004;351(26):2683-93.

4. Pope GD, Birkmeyer JD, Finlayson SR. National trends in utilization and in-hospital outcomes of bariatric surgery. J Gastrointest Surg. 2002;6(6):855-60.

5. Marceau P, Kaufman D, Biron S, Hould FS, Lebel S, Marceau S, et al. Outcome of pregnancies after biliopancreatic diversion. Obes Surg. 2004;14(3):318-24.

6. Bilenka B, Ben-Shlomo I, Cozacov C, Gold CH, Zohar S. Fertility, miscarriage and pregnancy after vertical banded gastroplasty operation for morbid obesity. Acta Obstet Gynecol Scand. 1995;74(1):42-4.

7. MacLean LD, Rhode BM, Shizgal HM. Nutrition following gastric operations for morbid obesity. Ann Surg. 1983;198(3):347-55.

8. Lima JG, Azevedo GD, Nobrega MLC, Maranhao TO, Nobrega LHC. Perfil menstrual de pacientes com obesidade mórbida. Arq Bras Endocrinol Metab. 2003;48(3 Suppl 2):S448.

9. Hood DD, Dewan DM. Anesthetic and obstetric outcome in morbidly obese parturients. Anesthesiology. 1993;79(6):1210-8.

10. Kakarla N, Dailey C, Marino T, Shikora SA, Chelmow D. Pregnancy after gastric bypass surgery and internal hernia formation. Obstet Gynecol. 2005; 105(5 Pt 2):1195-8.

11. Sheiner E, Levy A, Silverberg D, Menes TS, Levy I, Katz M, et al. Pregnancy after bariatric surgery is not associated with adverse perinatal outcome. Am J Obstet Gynecol. 2004;190(5):1335-40.

12. Wittgrove AC, Jester L, Wittgrove P, Clark GW. Pregnancy following gastric bypass for morbid obesity. Obes Surg. 1998;8(4):461-4.

13. Lam CM, Chow KM. Anemia in pregnancy. J Obstet Gynaecol Res. 2003;29(6):431.

14. Charles A, Domingo S, Goldfadden A, Fader J, Lampmann R, Mazzeo R. Small bowel ischemia after Roux-en-Y gastric bypass complicated by pregnancy: a case report. Am Surg. 2005;71(3):231-4. 